\title{
Simulation Research of Pipeline Flow Field Based on UDF under Different Open Valves
}

\author{
Yu-Xiang ZHANG ${ }^{1}$, Zhi-Jia LI ${ }^{1, a}$,Hui-Peng YAN ${ }^{1}$ and Yun-Teng MA ${ }^{1}$ \\ 1 Xi'an Research Inst. of Hi-Tech., Shanxi Xi'an, P. R. China
}

Keywords: Valve, UDF, Dynamic mesh, Flow field, FLUENT.

\begin{abstract}
Aiming at the manual ball valve in irrigation pump in industrial production pipeline, it is researched the flow characteristics of the valve and the pipeline under different way of opening valve. It is used user defined function (UDF) method and dynamic mesh technology of FLUENT software. Three valve opening states which are constant acceleration, constant velocity and constant deceleration are designed, and the valve opening rule of two-dimensional model for dynamic condition is simulated. The results show that the impact of constant acceleration rule of opening valve is smaller; its flow field is relatively stable; and it has less impact on the after the components of the pipeline.
\end{abstract}

\section{Introduction}

Valve controls the flow pressure, flow quantity and other flow parameters by changing the flow cross section, flow direction and others. It is a very important component in the liquid pipeline flow [1]. For irrigation pump system in some projects, in the process of full irrigation pump, it usually has a manual ball valve for manual adjustment, and other related parts are in the original state (pump blade rotating ignored under the impact to the structure), so its structure will not produce obvious influence on the flow field during the process of filling pump. Therefore, in order to control the flow velocity and flow rate in a reasonable range in the process of filling propellant to the pump, and don't have too much impact on the pump and liquid damper, it need to control the ball valve opening process with the certain rules.

Based on the above reasons, it needs to go into details of ball valve pipe flow field. Under the condition of different opening degree, the influence of the ball valve in the pipeline flow field is different. From the closing of the valve to open, impact of different way to open the valve also has a very profound influence. Due to valve structure is relatively complex, it's difficult to measure the flow field parameters; the related experimental research is more difficult to carry out; and the flow details of the flow field in pipeline cannot be fully grasped. Literature [2] has carried on the experimental study of flow noise for three kinds of valve (butterfly valve, ball valve, and gate valve). Literature [3] has simulated detailed flow structure in the flow field and the body stress distribution in switch process of four kinds of valves (gate valve, butterfly valve, ball valve, and regulating valve).

In this paper, it has explored a suitable method for the process of opening the valve according to the study of different laws of the valve opening and the changes of the flow field in irrigation pump pipeline.

\section{Physical Model of Ball Valve and Pretreatment}

In the industrial pump irrigation process, the reason of impact is mainly that gas in pipeline is affected by the impact of liquid flow rapidly to pump and the liquid damper during the irrigation pump in early. After all, liquid flows to the liquid damper, and the irrigation pump has been completed then, so the impact will not affect the normal work of the liquid propellant damper. In order to study the different valve opening process of gas flow in the pipeline, three kinds of the valve opening methods, which are constant acceleration, constant velocity and constant deceleration, have been studied. It has analysed that the flow state of different valve opening law, so as to find out a suitable valve control law [4]. 


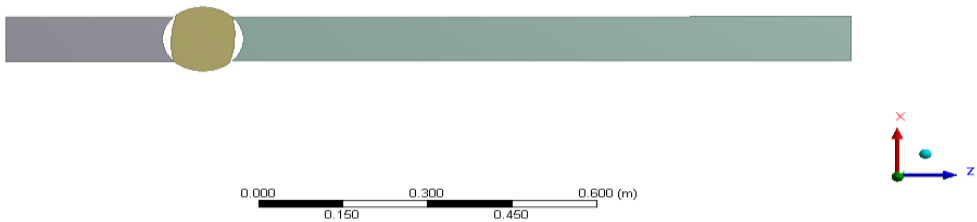

Figure 1. Two-dimensional physical model of ball valve and pipeline

In this paper, during the study of the valve opening methods, it has been used of two-dimensional pipeline and ball valve, thereby reducing the amount of calculation, and can better reflect the flow state of the pipeline. A two-dimensional physical model of the ball valve is established as shown in Figure1.

The diameter of the pipe is $100 \mathrm{~mm}$; the diameter of the valve core is $120 \mathrm{~mm}$; the length of the inlet pipeline is $0.25 \mathrm{~m}$; and the length of outlet pipeline is $1.25 \mathrm{~m}$, so that the gas flowing through the ball valve can be fully developed. Due to the finite element of software itself, in the beginning of the calculation, the valve must have a certain open degree, so that the initial state of the valve opening is 3 degrees, and it is supposed that the valve is closed at that time.

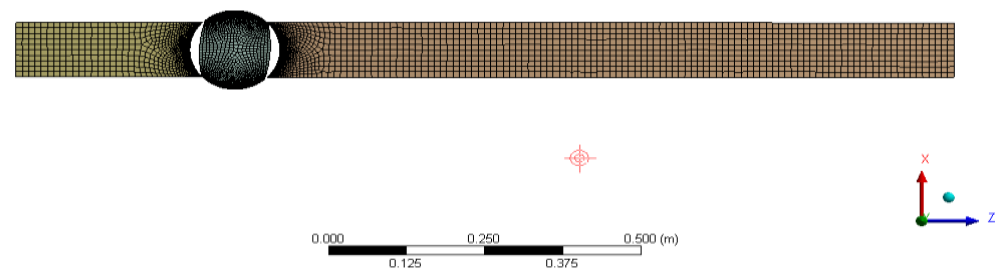

Figure 2. Mesh of ball valve model

Figure 2 shows the mesh of ball valve model. It is used the structured mesh; the overall mesh size is controlled to $2 \mathrm{~mm}$; the contact part of ball valve and the pipeline has locally dense, which the control mesh size is $1 \mathrm{~mm}$. Totally, the final amount of elements is 5963, and the number of nodes is 6387. This amount of mesh is conducive to the late dynamic mesh computing, and also can save the computing resources.

\section{Control Mode of The Ball Valve Opening}

In practical projects, it is not only to consider the impact of filling process, but also the working length of irrigation pump should be considered. As far as possible to reduce the irrigation time, it is required to develop the shorter the better. Considering the balance between the two, in this paper, it is set the constant acceleration and constant deceleration of the valve opening and closing angle acceleration are $1.2 \mathrm{rad} / \mathrm{s} 2$, and constant opening valve rate is $2.43 \mathrm{rad} / \mathrm{s}$. The whole length of the three law of the valve opening is $1.6 \mathrm{~s}$.

The three opening valve methods are under the same initial conditions and boundary conditions. Figure 3 is shown the initial moment of the ball valve pipeline pressure and velocity nephogram. The initial conditions of the pipeline pressure is 0 , and the flow velocity is also 0 . 


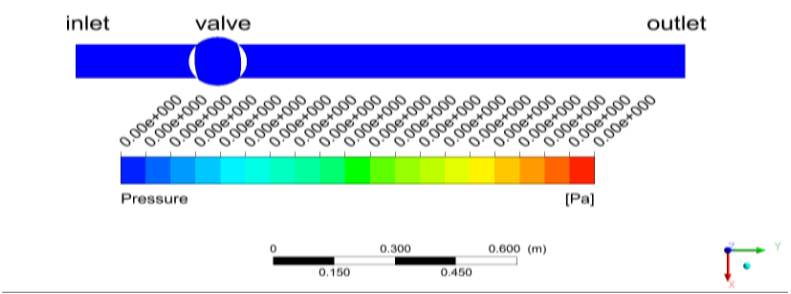

(a)

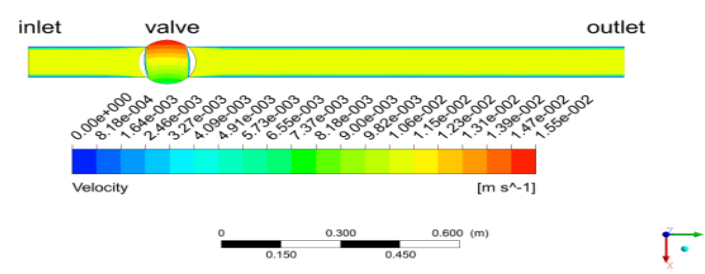

(b)

Figure 3. The pressure and velocity nephogram of the ball valve in the initial state

In FLUENT, the fluid material is set to air; the flow model is set to the standard k- $\varepsilon$ equation; and entrance velocity is $10 \mathrm{~m} / \mathrm{s}$. For the dynamic mesh part of the ball valve core, writing user defined function (UDF) and the dynamic mesh technology to control the valve core of the three kinds of motion laws [5]. The UDF of three kinds of different rules are as follows:

(1) Constant acceleration law:

\#include "udf.h"

DEFINE_ZONE_MOTION(fmotion,omega,axis,origin,velocity,time,dtime)

\{

if (time < 1.6)

$\{*$ omega $=1.2 *$ time; $\}$

else

$\{\quad *$ omega $=0.0 ; \quad\}$

N3V_D (velocity, $=, 0.0,0.0,0.0$ );

N3V_S( origin, $=, 0.0$ );

N3V_D(axis, =, 0.0,1.0,0.0);

return;

\}

(2) Constant velocity:

\#include "udf.h"

DEFINE_ZONE_MOTION(fmotion,omega,axis,origin,velocity,time,dtime)

\{

if (time < 1.6)

\{ *omega $=2.429498 ;$ \}

else

$\{\quad *$ omega $=0.0 ;\}$

N3V_D (velocity, $=, 0.0,0.0,0.0$ );

N3V_S(origin, $=, 0.0$ );

N3V_D(axis, =, 0.0,1.0,0.0);

return;

\}

(3) Constant deceleration:

\#include "udf.h"

DEFINE_ZONE_MOTION(fmotion,omega,axis,origin,velocity,time,dtime)

\{

if (time < 1.6)

$\{$ *omega $=1.92-1.2 *$ time; $\}$

else

$\{\quad *$ omega $=0.0 ;\}$

N3V_D (velocity, $=, 0.0,0.0,0.0)$;

N3V_S(origin, $=, 0.0$ );

N3V_D(axis,=,0.0,1.0,0.0); 
return;

\}

The calculation time step is $0.05 \mathrm{~s}$, and the total flow time is $3 \mathrm{~s}$.

\section{Flow Field Result Analysis [6]}

The flow fields of three kinds of valve opening rules are compared. Respectively, (a), (b) and (c) of Figure 4 - Figure 8 are the pressure nephogram under constant acceleration, constant velocity and constant deceleration states. The length under the three laws of the open valve is $1.6 \mathrm{~s}$, but there are still great differences at the same time before and after the valve motion in the flow state.

Analyzed Figure 4, Figure 5 and Figure 6 flowing from $0.4 \mathrm{~s}$ to $2.0 \mathrm{~s}$, the constant acceleration valve is opened slowest, and under the condition of constant deceleration valve opens the fastest; at about $1.6 \mathrm{~s}$, the three are almost completely open at the same time, since then, the gas flow is equivalent to the same pipe flow state.

Compared with $0.4 \mathrm{~s}, 0.8 \mathrm{~s}$ and $1.2 \mathrm{~s}$, each moment of the pressure nephogram. In general, the change of pressure nephogram of constant velocity opening valve under the flow state is the smallest, and the ball valve has a small pressure difference before and after; inside the valve core it has some pressure gradient and the pressure of the vacuum area. Therefore in the condition of constant velocity opening valve, the pressure inside the pipeline field basically performs into three parts: respectively is positive pressure part before the valve, negative pressure part after and the valve core. Constant accelerating state is the most stable in the three flow pattern. From the beginning to the final, the pressure gradient is small, and the pressure is relatively stable. Outlet pressure of constant deceleration gradually changes in the flow process. After the ball valve, the pipeline pressure is negative at first, which is due to the previous valve resistance and the inertia of gas flow, thereby forming a partial vacuum in the valve outlet area. After a certain distance, the velocity decreases and the pressure gradually returned to normal; the pipeline pressure changes from small to big; it reached the outside atmospheric pressure as set at the outlet, which is consistent with the actual situation.

Taking into account the disorder degree of the pressure of the outlet pipe, constant velocity or constant acceleration should be used in the design at the preliminary judgment, so as to make the pipeline pressure in a more stable state after the ball valve.

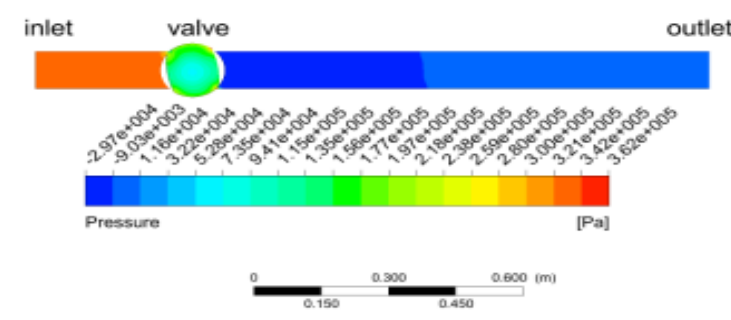

(a)

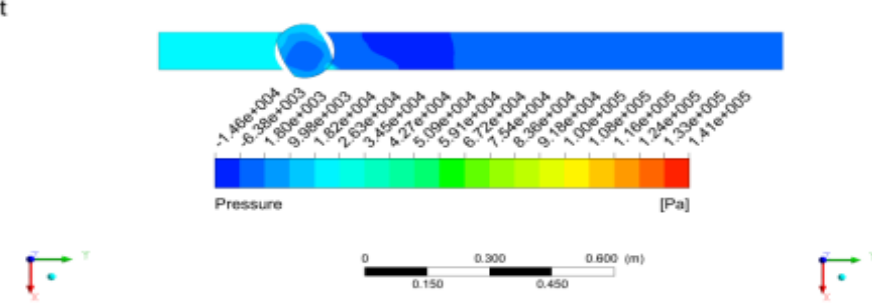

(b)

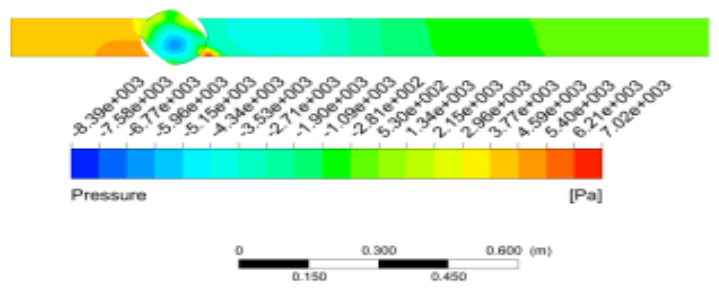

(c)

Figure $4.0 .4 \mathrm{~s}$ constant acceleration, constant velocity and constant deceleration opening valve pressure nephogram 


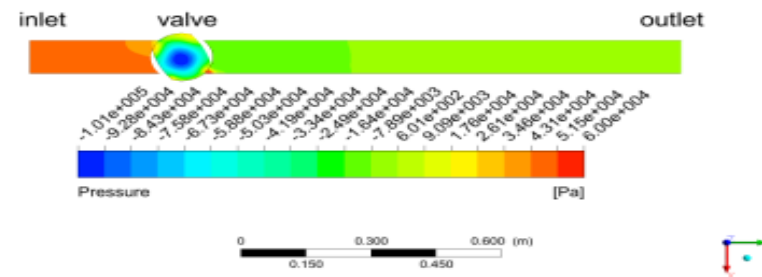

(a)

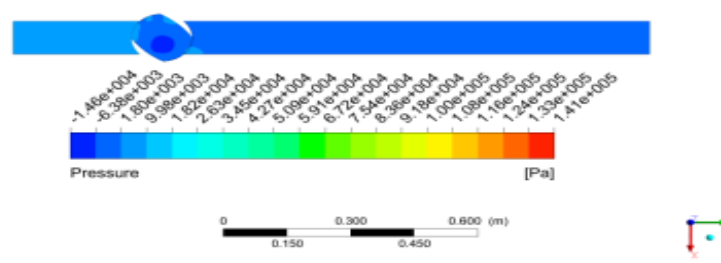

(b)

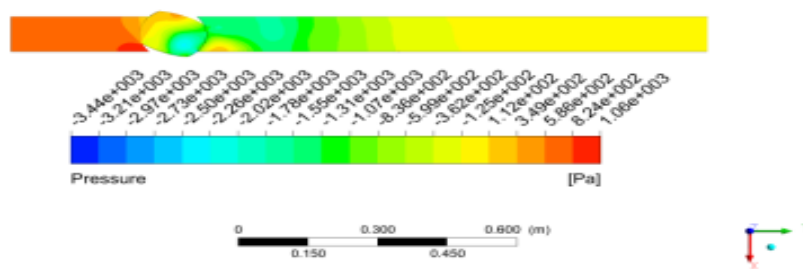

(c)

Figure 5. 0.8s Constant acceleration, constant velocity and constant deceleration opening valve pressure

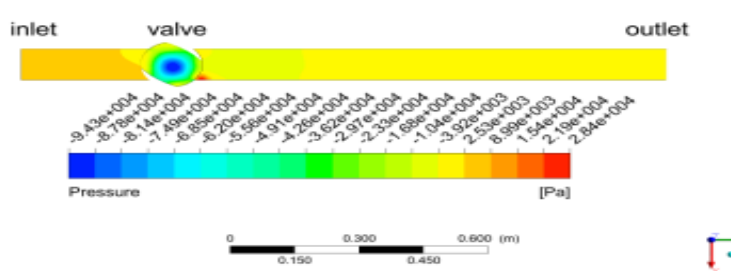

(a)

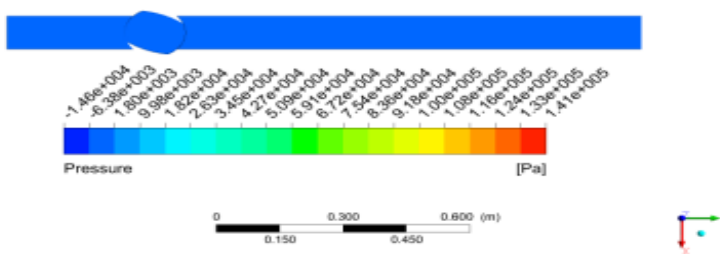

(b)

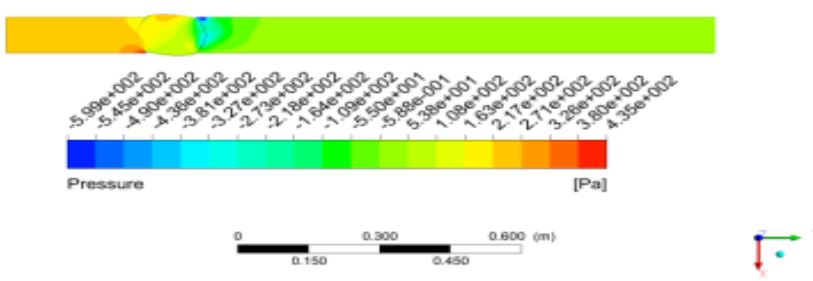

(c)

Figure 6. 1.2s Constant acceleration, constant velocity and constant deceleration opening valve pressure nephogram

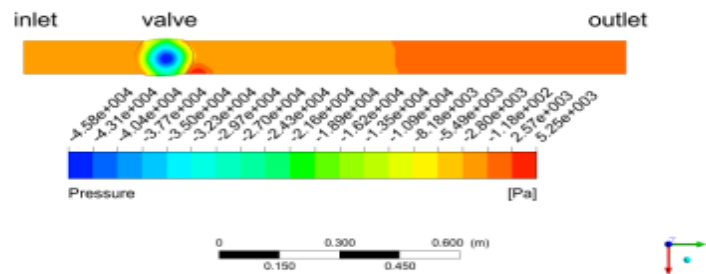

(a)

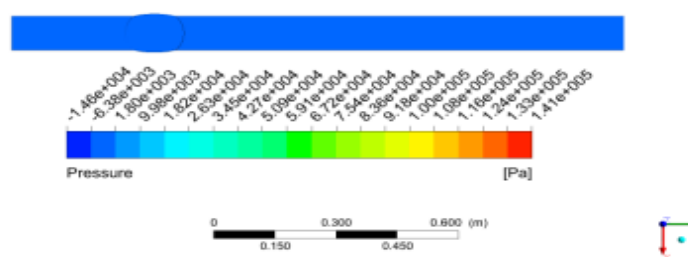

(b)

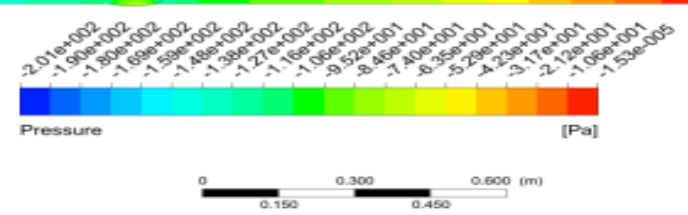

(c)

Figure 7. 1.6s Constant acceleration, constant velocity and constant deceleration opening valve pressure 


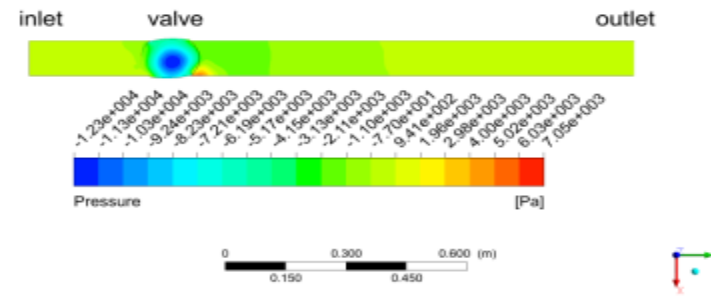

(a)

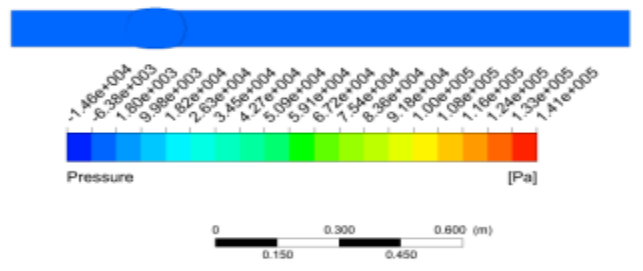

(b)

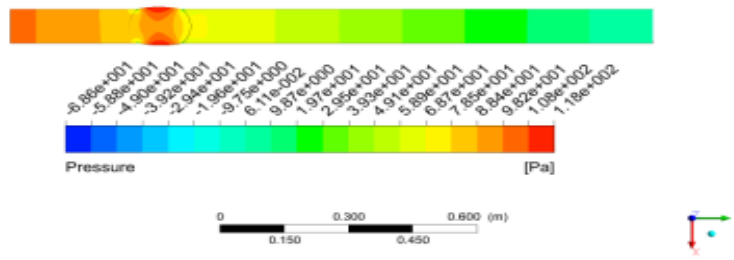

(c)

Figure 8. 2.0s constant acceleration, constant velocity and constant deceleration opening valve pressure nephogram

Figure 9-13 is the velocity nephogram of three kinds of open valve state and the pipeline. The initial conditions and boundary conditions, the way of opening the valve are all the same as the state of the pressure analysis. Compared the different moment of each valve open way, the overall flow velocity will be increased gradually along with the gradually opening of the ball valve. For different ways of opening the valve at the same time, the ball valve opening is different in most cases, and the bigger the valve opening, the flow velocity is relatively larger. In the process of opening the valve, due to the effect of damping of the valve core, the effective cross-sectional area of fluid flow through the valve core is also changing. In the outlet position of the ball valve, the flow velocity which is connected to the ball valve core is significantly higher than area that is not connected to the valve core. So there is speed stratification in the export pipeline, and the relative area size of the high speed area and the low speed region also shows the opening size of the ball valve.

Analyzed the valve opening of $0.2 \mathrm{~s}$ to $1.6 \mathrm{~s}$ state, the flow velocity of constant acceleration state is gradually smooth transited to the lager velocity from the initial small opening speed. The valve fully opened after 1.6s, and the velocity retreats, but the appearance of the speed increased steadily overall. In constant deceleration opening valve state, initial valve opening quickly, and thus the performance of the initial flow rate rises rapidly, and soon reached the peak velocity. When the ball valve is about to a fully open, it gradually slows to open. In this state, the flow velocity no longer increased, and it will retreat to the normal value. The opening velocity of constant velocity state is opened faster than the acceleration and slower than the deceleration state at first. So the valve opening process is also a part of the original high speed region and low speed area. The high speed region performs closer to the flow field of constant deceleration state, and they have a similar flow pattern.

From 1.6s and 2.0s, when the valve is fully open after the influence of the valve opening, the flow state in pipeline is still not very smooth. After each kind of thee open rule, the pipeline is retained the original flow field characteristics in a certain extent.

Compared with the different flow field in this condition, open flow valve after the constant acceleration is the most stable. The most complex area of the pipeline is in the valve core. After the valve core, pipeline outlet position is relatively stable. Compared with the states of constant velocity and constant deceleration, the velocity gradient is not so obvious. 


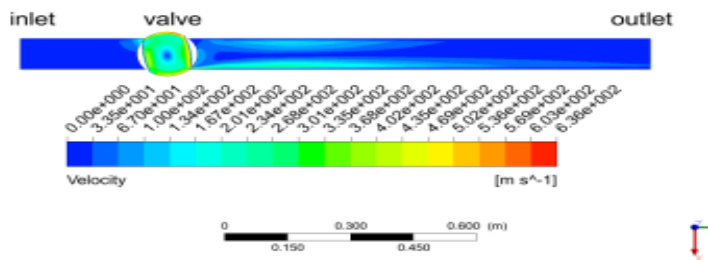

(a)

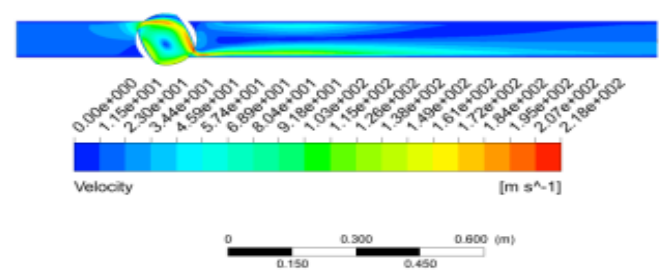

(b)

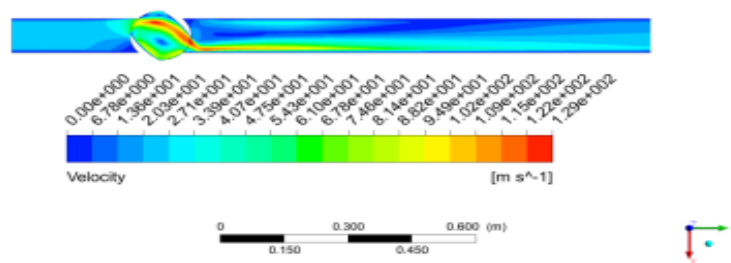

(c)

Figure 9. $0.4 \mathrm{~s}$ constant acceleration, constant velocity and constant deceleration opening valve velocity nephogram

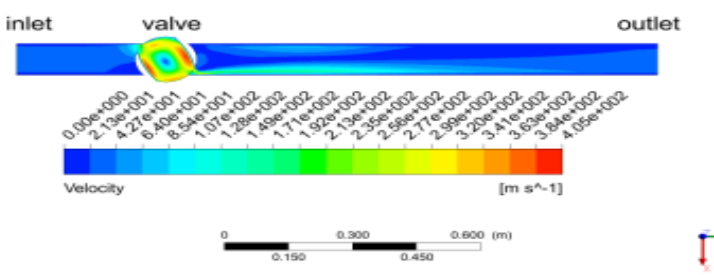

(a)

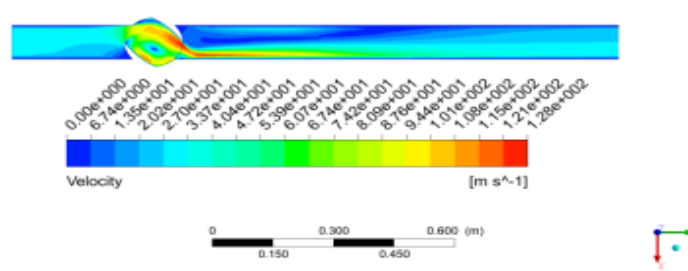

(b)

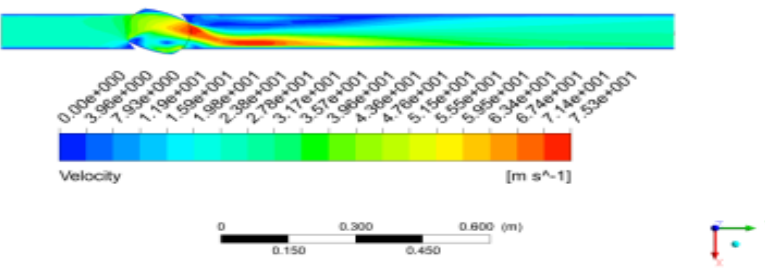

(c)

Figure 10. 0.8 s constant acceleration, constant velocity and constant deceleration opening valve velocity nephogram

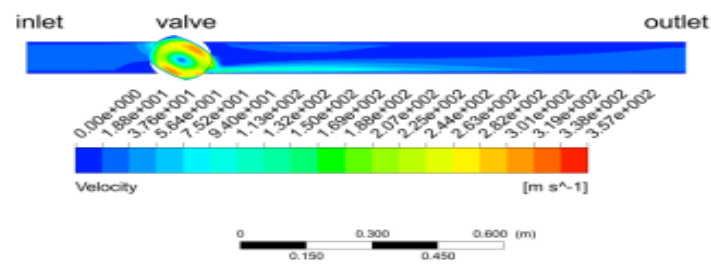

(a)
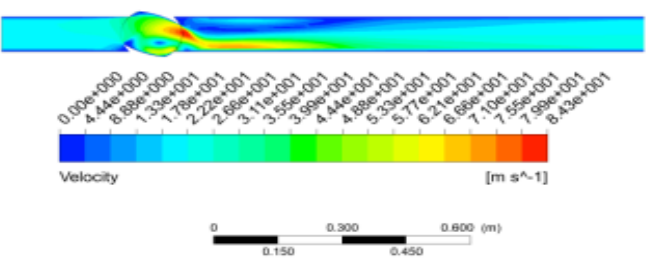

(b)

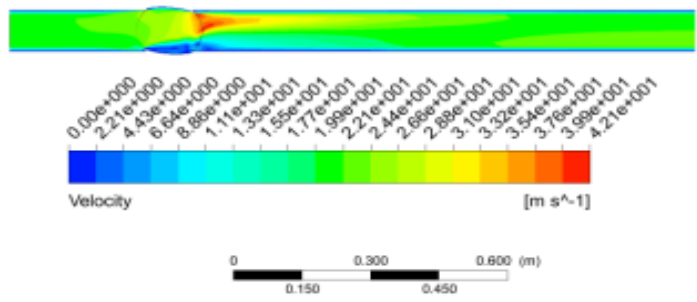

(c)

Figure 11. 1.2s constant acceleration, constant velocity and constant deceleration opening valve velocity nephogram 


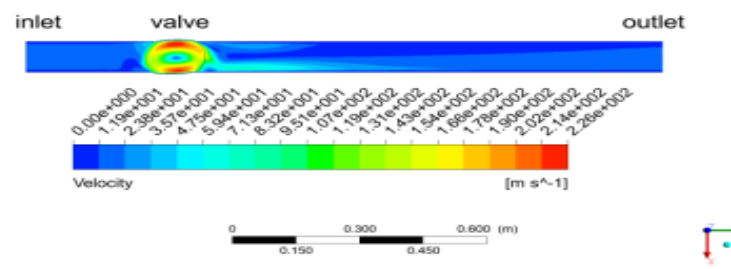

(a)

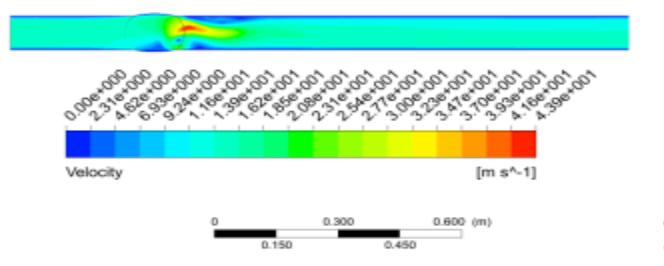

(b)

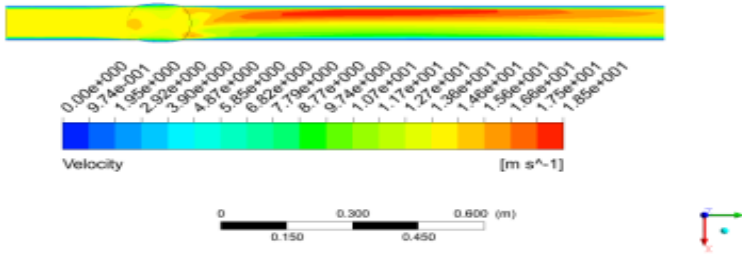

(c)

Figure 12. 1.6s constant acceleration, constant velocity and constant deceleration opening valve velocity nephogram

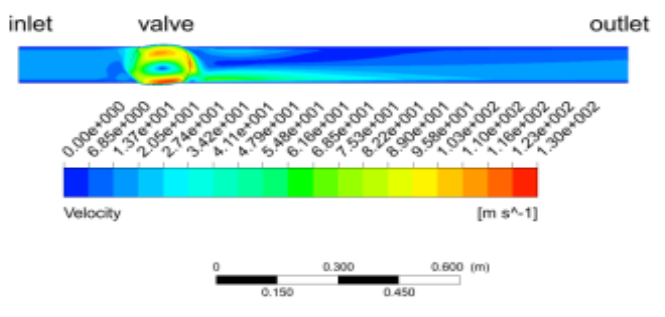

(a)

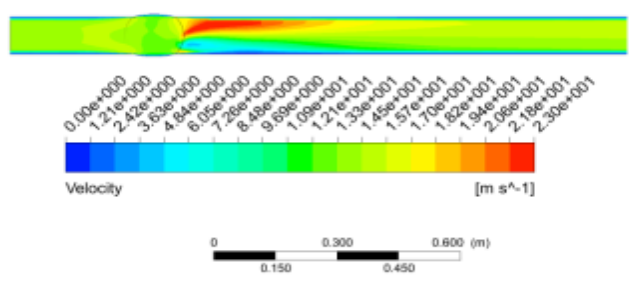

(b)

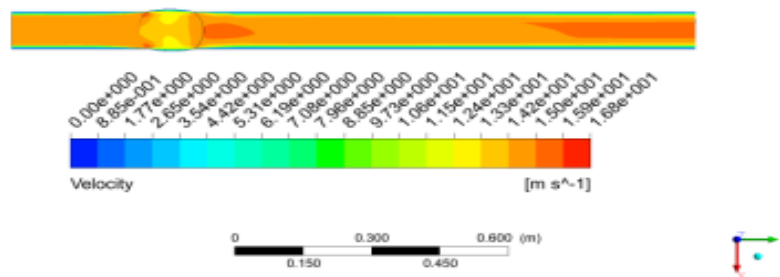

(c)

Figure 13. 2.0s constant acceleration, constant velocity and constant deceleration opening valve velocity nephogram

\section{Conclusions}

(1)According to the above research on the opening methods of the valve pipeline, for the irrigation pump process, the change of initial state of the flow field is very intense. Therefore, the different law of open valve on the flow field in the pipeline has a great influence.

(2)Considered the pressure field and velocity field, the impact for flow field of the open valve with constant acceleration is less, and the flow field in the pipeline is more stable. So that it is suitable to be used in valve opening.

\section{Acknowledgements}

This work was financially supported by The National Natural Science Fund (51275517).

\section{References}

[1] H.Y. Zhang, Z.R. Yan, Y.F. Chen, etc. Design and selection of ball valve. (1994). 
[2] S. Wu, W.P. Zhang. Numerical simulation of valve flow field and experimental study of flow noise [J]. Valve, 1 (2005) 7-10.

[3] H.P. Liu, F. Chen, B. Ma. Numerical Simulation of Flow Field in the Valve Based on the Moving Mesh and the UDF [J]. Turbine Technology, 50,2(2008): 106-108.

[4] G.Z. Zhang. Fluid Numerical Analysis in Feed-water System Pipes of the Super Critical Power Unit [D]. Wuhan: Master's degree thesis, Huazhong University of Science and Technology, (2007)51-57.

[5] H.T. Sui, P.F. Li, S.H. Ma, etc. Proficient in CFD Dynamic Mesh Engineering Simulation and Case Study (2013)219-225.

[6] K. Shi. Transient Simulation and Experimental Study on the Opening Process of Ball Valve [D]. Zhejiang: Master Thesis of Zhejiang Sci-Tech University, (2014)23-47. 